\title{
Metal sorption onto Environmental NanoPlastics (ENPs), towards a mechanistic comprehension?
}

\author{
BLANCHO FLORENT ${ }^{1}$, MELANIE DAVRANCHE ${ }^{1}$ AND \\ JULIEN GIGAULT ${ }^{2}$ \\ ${ }^{1}$ Univ Rennes, CNRS, Géosciences Rennes, UMR 6118 \\ ${ }^{2}$ University of Laval \\ Presenting Author: blancho.florent@gmail.com
}

Recently, a large scientific interest was given to nanoplastics regarding major environmental issues. Despite different scientific approaches to study their behavior and impacts, a guiding theme was to produce/use adequate models [1-3]. Applied to metal sorption, such guidance lead to questioning the use of these actual models as they do not present environmentally relevant shape and surfaces properties [1-3]. In response, the use of the altered layer of plastics debris to produce environmental nanoplastics (ENPs) were proposed [4]. Specific plastics debris remained for decades at the ocean surface which result to the production of an altered layer at their surface. This oxidized layer can be considered as environmentally relevant as it was weathered under environmental conditions. Within this approach, Davranche et al. 2019 [4], reported $\mathrm{pH}$ dependent $\mathrm{Pb}$ sorption by these ENPs. Such behavior can be explained through the sorption onto (de)protonable functional groups (i.e. hydroxyl, phenolic, carboxylic, etc.). However actually the metal-surface relationship description is restricted to an empirical relationship, since, knowledge about the physico-chemical surface properties of nanoplastics remains limited. Our aim, here, is to use a mechanistic approach to describe nanoplastic metal sorption properties. ENPs were produced in large amount using a revisited protocol based on an extra extraction step and the removing of associated organic matter. Specific surface area (BET method), Carbon bonding (XPS) and acidic surface properties (potentiometric titration) were determined. Isotherm and $\mathrm{pH}$-edge of Lead adsorption were performed. Finally, experimental data were used to model $\mathrm{Pb}$ adsorption by ENPs and thus decipher the metal sorption mechanism occurring at their surface. Major results are consisted of a (i) detailed physico-chemical description of the ENPs surface and (ii) the description of the mechanisms and parameters that control the metal adsorption by nanoplastics. Such results provide primordial assets to predict/understand impacts of nanoplastics in the environment and major associated issues as well.

[1] El Hadri et al. (2020), NanoImpact, 17, 100206.

[2] Pessoni et al. (2019), Environ. Sci.: Nano, 6 (7)

[3] Mitrano et al. (2019), Nature Nanotechnology, 14, 362368

[4] Davranche et al. (2019), Environmental Pollution, 249, 940-948 